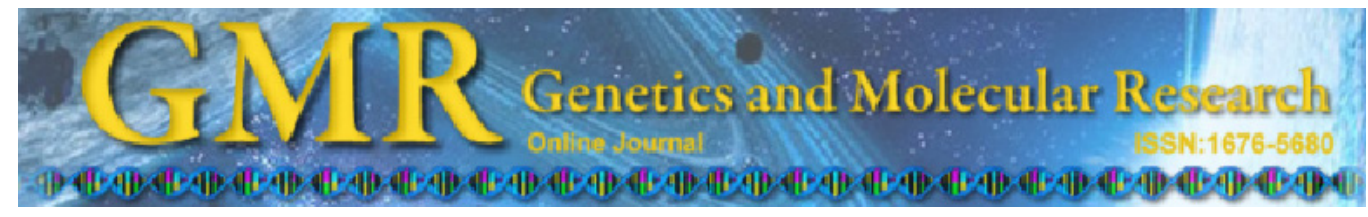

\title{
Isolation and characterization of 24 polymorphic microsatellite loci in Sepioteuthis lessoniana
}

\author{
X.-D. Zheng ${ }^{1,2}$, Z.-R. Zuo ${ }^{1}$, X.-J. Su ${ }^{3}$ and Q. $\mathrm{Li}^{1}$ \\ ${ }^{1}$ Fisheries College, Ocean University of China, Qingdao, China \\ ${ }^{2}$ Institute of Evolution and Marine Biodiversity, \\ Ocean University of China, Qingdao, China \\ ${ }^{3}$ College of Animal Science and Technology, Guangxi University, \\ Nanning, Guangxi, China \\ Corresponding author: X.-D. Zheng \\ E-mail: xdzheng@ouc.edu.cn
}

Genet. Mol. Res. 11 (4): 3961-3965 (2012)

Received July 4, 2012

Accepted September 21, 2012

Published November 14, 2012

DOI http://dx.doi.org/10.4238/2012.November.14.3

\begin{abstract}
Owing to their codominant, multiallelic, and highly polymorphic nature, microsatellite markers have been used widely in population genetics and biological resource conservation studies. To investigate the genetic structure of Sepioteuthis lessoniana, we developed 24 microsatellite DNA markers and assessed the polymorphism of each locus in a wild $S$. lessoniana population. The number of alleles per locus ranged from 4 to 26, and the observed and expected heterozygosities varied from 0.188 to 1.000 and 0.392 to 0.959 with an average of 0.675 and 0.852 , respectively. These microsatellite loci will be useful tools in future studies of population genetic structure in this species.
\end{abstract}

Key words: Microsatellite; Cephalopoda; Sepioteuthis lessoniana; Enriched genomic library 


\section{INTRODUCTION}

Bigfin reef squid, Sepioteuthis lessoniana (Lesson, 1830), are marine animals found from the shores from the Hawaiian Islands to the tropical Pacific Ocean and the Red Sea. They are commonly mistaken as cuttlefish, but they are indeed a completely different species. S. lessoniana has been placed within the Sepioteuthis branch in the cephalopod phylogenetic tree based on mitochondrial gene arrangement (Akasaki et al., 2006). Allozyme electrophoresis (Triantafillos and Adams, 2005) has been used to investigate the taxonomic status of northern calamari, and results indicate that $S$. lessoniana most likely comprises two "cryptic" biological species in Shark Bay, western Australia.

The only previous genetic investigations undertaken on $S$. lessoniana have demonstrated three taxa in the waters around Japan based on allozyme profiles (Izuka et al., 1994, 1996). Cryptic or sibling species are those not recognized a priori on morphological grounds and are a relatively common phenomenon among marine invertebrate groups (Knowlton, 1993; Thorpe et al., 2000). Otherwise cryptic species do exist in loliginid squid in the region (Yeatman and Benzie, 1994); therefore, a need has arisen to resolve the species status of $S$. lessoniana, preferably with molecular genetic techniques.

Owing to their codominant, multiallelic, and highly polymorphic nature, microsatellite markers have been used widely in population genetics and biological resource conservation research. Herein we present the first polymorphic microsatellite markers for S. lessoniana. These markers will be useful in population genetic studies and the conservation of this species.

\section{MATERIAL AND METHODS}

One live S. lessoniana was sampled from Lingshui, Hainan Province, China $\left(18.48^{\circ} \mathrm{N}\right.$, $110.02^{\circ} \mathrm{E}$ ). Genomic DNA was isolated from mantle muscle and subsequently digested with $\mathrm{MboI}$. DNA fragments were ligated to oligonucleotide adapters (Gardner et al., 1999). We used electrophoresis on a $2 \%$ NuSieve GTG (Cambrex, USA) agarose gel to isolate small fragments (350$1000 \mathrm{bp})$. Biotin-labeled dinucleotide repeat sequences $\left[(\mathrm{CA})_{15}\right]$ were hybridized to these DNA fragments, and the hybrid mixture was incubated with streptavidin-coated magnetic spheres (Promega, USA). After washing to remove the non-simple sequence repeat (SSR) fragments, the eluted single-stranded DNA contained the selected microsatellite DNA. As the primer, adapter A was used to amplify the selected DNA fragments using hot start polymerase chain reaction (PCR).

The amplified DNA fragments were ligated with pMD19-T plasmid vectors (TaKaRa, Japan) and the complexes were transformed into Escherichia coli DH5a competent cells (Toyobo, Japan). White clones were PCR screened with two vector primers and a non-biotin-labeled (CA) primer. Screening amplifications were performed as follows: $94^{\circ} \mathrm{C}$ for $3 \mathrm{~min}$ followed by 35 cycles of $94^{\circ} \mathrm{C}$ for $45 \mathrm{~s}, 56^{\circ} \mathrm{C}$ for $45 \mathrm{~s}$, and $72^{\circ} \mathrm{C}$ for $45 \mathrm{~s}$, and a final extension of $5 \mathrm{~min}$ at $72^{\circ} \mathrm{C}$.

We selected 360 clones for amplification, 97 of which were isolated for sequencing using a BigDye Terminator Cycle sequencing kit and an ABI PRISM 3730 Genetic Analyser (Applied Biosystems). Screening by the SSR Hunter 1.3 software (Li and Wan, 2005) showed that 80 sequences contained microsatellites with at least four uninterrupted repeats. With the exception of the biotin-labeled AC SSR probes, TC, AG, AT, AAG, AGT, ACAG, AGAT, ATAG, AAAG, and AGAA occurred in the microsatellites, which suggested that this probe made a positive contribution to the creation of the SSR-enrichment library from the genomes. All PCR primers were designed with PRIMER 5 (http:// www.premierbiosoft.com/). We discarded the hybrid clones, duplicates and those sequences with short unique regions flanking the microsatellite array. In total, 52 PCR primer pairs were designed. 
We used polyacrylamide gel electrophoresis to test the primer pairs in $30 \mathrm{~S}$. lessoniana individuals sampled from Lingshui, Hainan Province. PCR amplifications were carried out in $10-\mu \mathrm{L}$ volumes containing $0.25 \mathrm{U}$ Taq DNA polymerase (TaKaRa), 1X PCR buffer, $0.2 \mathrm{mM}$ deoxyribonucleotide triphosphate mix, $1 \mu \mathrm{M}$ each primer set, $1.5 \mathrm{mM} \mathrm{MgCl}$, and approximately 100 ng template DNA. The PCR thermal conditions were as follows: 3 min at $94^{\circ} \mathrm{C}$, followed by

\begin{tabular}{|c|c|c|c|c|c|c|c|c|c|}
\hline Locus & $\begin{array}{l}\text { Accession } \\
\text { No. }\end{array}$ & Repeat motif & Primer sequence $\left(5^{\prime}-3^{\prime}\right)$ & $\mathrm{Ta}\left({ }^{\circ} \mathrm{C}\right)$ & $\begin{array}{l}\text { No. of } \\
\text { alleles }\end{array}$ & $\begin{array}{c}\text { Size } \\
\text { range (bp) }\end{array}$ & $H_{\mathrm{o}}$ & $H_{\mathrm{E}}$ & $\mathrm{P}$ \\
\hline Sle02 & JQ696966 & $\begin{array}{l}(\mathrm{CT})_{5}(\mathrm{CT})_{7}(\mathrm{CT})_{7} \\
(\mathrm{TTTC})_{6}\end{array}$ & $\begin{array}{l}\text { TCTACGGCTACATTTACCTTT } \\
\text { GGGGTGAGCATTTGAGTAAG }\end{array}$ & 56 & 16 & $311-379$ & 0.656 & 0.921 & $0.0000^{*}$ \\
\hline Sle 03 & JQ696967 & $\begin{array}{l}(\mathrm{TG})_{25}(\mathrm{AG})_{5} \\
(\mathrm{AG})(\mathrm{AG})\end{array}$ & $\begin{array}{l}\text { AAGGCGAAGTAATACAGA } \\
\text { CACTTCTTAGTCCCTCT }\end{array}$ & 56 & 15 & $179-221$ & 0.807 & 0.813 & 0.5101 \\
\hline Sle04 & JQ696968 & $(\mathrm{CT})_{9}(\mathrm{CA})_{14}(\mathrm{CA})_{7}$ & $\begin{array}{l}\text { CTCCCTCACATCTATTACCA } \\
\text { GAGAGCAAGATGAAAGTGAAG }\end{array}$ & 56 & 13 & 204-232 & 1.000 & 0.893 & 0.0350 \\
\hline Sle06 & JQ696969 & $\begin{array}{l}(\mathrm{GA})_{4}(\mathrm{AG})_{5} \\
(\mathrm{GT})_{20}(\mathrm{GA})_{44}\end{array}$ & $\begin{array}{l}\text { TGTACCAAGTGTTATCTCCG } \\
\text { GTTGCTATCAGTTTCTGTTAGTC }\end{array}$ & 56 & 16 & $273-329$ & 0.688 & 0.931 & $0.0000 *$ \\
\hline Sle07 & JQ696970 & $\begin{array}{l}(\mathrm{TG})_{6}(\mathrm{GA})_{4}(\mathrm{GA})_{4} \\
(\mathrm{TG})_{4}(\mathrm{GA})_{5}(\mathrm{GA})_{4}\end{array}$ & $\begin{array}{l}\text { AGCGTGCATGAGAAGGAA } \\
\text { CTGCAATGGGCTTGTTTAC }\end{array}$ & 56 & 8 & $235-265$ & 0.625 & 0.741 & $0.0000^{*}$ \\
\hline Sle09 & JQ696971 & $\begin{array}{l}(\mathrm{AG})_{8}(\mathrm{GT})_{12} \\
(\mathrm{AG})_{20}(\mathrm{AG})_{4}\end{array}$ & $\begin{array}{l}\text { GTGTTAAAAGGTCCACTAAAG } \\
\text { GCCCACCCATAAATAAATAC }\end{array}$ & 62 & 13 & $276-334$ & 0.633 & 0.906 & 0.0004 \\
\hline Sle10 & JQ696972 & $(\mathrm{AC})_{7}(\mathrm{AT})_{4}$ & $\begin{array}{l}\text { TATGCTACAAAACCAAAC } \\
\text { ATATTCCACCTTGAAAAC }\end{array}$ & 56 & 4 & $275-281$ & 0.188 & 0.392 & 0.0006 \\
\hline Sle11 & JQ696973 & $\begin{array}{l}(\mathrm{TG})_{5}(\mathrm{TG})_{9}(\mathrm{TG})_{8} \\
(\mathrm{GC})_{4}(\mathrm{CT})_{1}(\mathrm{TG})_{3}\end{array}$ & $\begin{array}{l}\text { GCTATAATACGCTCAAGTACCGA } \\
\text { CAGCTATCTCAGCCACCAAA }\end{array}$ & 62 & 16 & $348-408$ & 0.625 & 0.923 & $0.0000^{*}$ \\
\hline Sle12 & JQ696974 & $(\mathrm{AAAG})_{6}(\mathrm{AGAA})_{14}$ & $\begin{array}{l}\text { GCCTATTTATTACATTGGTCG } \\
\text { TTTTACAACTTCACTCTTCCTTT }\end{array}$ & 58 & 26 & $168-284$ & 0.968 & 0.959 & 0.1816 \\
\hline Sle15 & JQ696975 & $(\mathrm{AC})_{31}$ & $\begin{array}{l}\text { ACGAGAAGTTGAGTTATCTGAAA } \\
\text { AACTGAAGTAAATTGGTAAATCAG }\end{array}$ & 60 & 14 & $193-233$ & 0.724 & 0.893 & $0.0000 *$ \\
\hline Sle16 & JQ696976 & $(\mathrm{AGT})_{9}$ & $\begin{array}{l}\text { TCGCACAACACCAGCATAGAG } \\
\text { CGTCCGATTCACCGCTTACT }\end{array}$ & 60 & 8 & $120-144$ & 0.903 & 0.800 & 0.0293 \\
\hline Sle17 & JQ696977 & $(\mathrm{GC})_{5}(\mathrm{GT})_{16}$ & $\begin{array}{l}\text { CAGTAAGCGGTGAATCGGAC } \\
\text { AGTGCGTATGCGTACCCTTT }\end{array}$ & 60 & 21 & $126-192$ & 0.967 & 0.945 & 0.0626 \\
\hline Sle22 & JQ696978 & $(\mathrm{TC})_{9}(\mathrm{CA})_{27}(\mathrm{TC})_{12}$ & $\begin{array}{l}\text { CACAGTACAGCAAGGATTTCT } \\
\text { CTGTCAGTCGGTGAGTTTGT }\end{array}$ & 60 & 11 & $229-281$ & 0.875 & 0.875 & 0.0262 \\
\hline Sle24 & JQ696979 & $(\mathrm{CT})_{8}(\mathrm{TC})_{4}(\mathrm{CT})_{4}$ & $\begin{array}{l}\text { TCTGTTTCTTTATTCCTACCTCT } \\
\text { GGGATTGTTCCAGTTGAGC }\end{array}$ & 60 & 4 & $275-287$ & 0.344 & 0.597 & $0.0000 *$ \\
\hline Sle25 & JQ696980 & $(\mathrm{AGAT})_{16}$ & $\begin{array}{l}\text { GATCGAAACATTTGAGCACT } \\
\text { ATTTCCCATTCTGTCTTTGT }\end{array}$ & 56 & 13 & $130-180$ & 0.406 & 0.904 & $0.0000^{*}$ \\
\hline Sle29 & JQ696981 & $(\mathrm{ACAG})_{5}(\mathrm{GT})_{27}$ & $\begin{array}{l}\text { GAAAGACGCCAAGAGCATC } \\
\text { ATTTCGTTTCGTTACCGTTG }\end{array}$ & 62 & 19 & $270-314$ & 1.000 & 0.942 & 0.4457 \\
\hline Sle31 & JQ696982 & $(\mathrm{TG})_{14}(\mathrm{GT})_{37}$ & $\begin{array}{l}\text { GCCGTTGACTGATATGCTAA } \\
\text { GTGGGACGTTTGTCGTTT }\end{array}$ & 62 & 12 & $342-378$ & 0.548 & 0.915 & $0.0000^{*}$ \\
\hline Sle32 & JQ696983 & $\begin{array}{l}(\mathrm{GA})_{6}(\mathrm{ATAG})_{4} \\
\left.(\mathrm{AG})_{16}(\mathrm{GT})_{26} \mathrm{AG}\right)_{5}\end{array}$ & $\begin{array}{l}\text { GCCGAAATGAAGATGAGA } \\
\text { CGTCTCCTCCTCACTTACTC }\end{array}$ & 56 & 17 & $258-314$ & 0.833 & 0.934 & 0.0024 \\
\hline Sle33 & JQ696984 & $(\mathrm{CA})_{25}$ & $\begin{array}{l}\text { CATCGTTGCCGTGTAGTCT } \\
\text { TGATGTCCTTTCCATATCACTA }\end{array}$ & 56 & 15 & $292-330$ & 0.828 & 0.921 & 0.2520 \\
\hline Sle36 & JQ696985 & $\begin{array}{l}(\mathrm{GA})_{20}(\mathrm{GA})_{4}(\mathrm{TG})_{6} \\
(\mathrm{TG})_{6}(\mathrm{TG})_{5}(\mathrm{TG})_{5}\end{array}$ & $\begin{array}{l}\text { CATCCGATTATCACTGCCT } \\
\text { TTCCCGATATTTCTATTACTCA }\end{array}$ & 60 & 17 & $305-347$ & 0.367 & 0.928 & $0.0000 *$ \\
\hline Sle37 & JQ696986 & $\begin{array}{l}(\mathrm{GA})_{12}(\mathrm{GT})_{11}(\mathrm{GT})_{11} \\
(\mathrm{GA})_{8}(\mathrm{AG})_{4}\end{array}$ & $\begin{array}{l}\text { AGTCAGTCTGTCAGTCGGTG } \\
\text { CAGTACAGCAAGGATTTCTCA }\end{array}$ & 60 & 8 & $267-287$ & 0.519 & 0.806 & 0.0067 \\
\hline Sle41 & JQ696987 & $(\mathrm{AAG})_{15}$ & $\begin{array}{l}\text { ACCCGAGGAGACTTACTATCACTC } \\
\text { TGTTGTTGCTGGCTGCTCT }\end{array}$ & 56 & 12 & $222-270$ & 0.308 & 0.910 & $0.0000 *$ \\
\hline Sle43 & JQ696988 & $\begin{array}{l}(\mathrm{GT})_{7}(\mathrm{GA})_{9}(\mathrm{AG})_{5} \\
(\mathrm{GA})_{4}(\mathrm{GA})_{8}(\mathrm{GT})_{5}(\mathrm{GA})_{6} \\
(\mathrm{AG})_{7}(\mathrm{GA})_{6}(\mathrm{AG})_{5} \\
(\mathrm{GA})_{6}(\mathrm{GA})_{10}(\mathrm{GA})_{8}\end{array}$ & $\begin{array}{l}\text { AGGGACAGATGAAGAAGGT } \\
\text { CGTCTTGATAAAACAGCGT }\end{array}$ & 60 & 9 & $295-365$ & 0.581 & 0.760 & 0.0091 \\
\hline Sle 48 & JQ696989 & $\begin{array}{l}(\mathrm{GT})_{10}(\mathrm{GA})_{12}(\mathrm{AG})_{10} \\
(\mathrm{GA})_{4}(\mathrm{GA})_{4}(\mathrm{TG})_{11}(\mathrm{TG})_{7} \\
(\mathrm{GA})_{5}(\mathrm{AG})_{4}(\mathrm{AG})_{4} \\
(\mathrm{GA})_{10}(\mathrm{TAGA})_{4}\end{array}$ & $\begin{array}{l}\text { AGAAGCAAGGAGAAATATAGACAG } \\
\text { AACATTCCAACCAAAAGAGG }\end{array}$ & 60 & 11 & $352-394$ & 0.813 & 0.842 & 0.0965 \\
\hline
\end{tabular}


35 cycles of $45 \mathrm{~s}$ at $94^{\circ} \mathrm{C}, 45 \mathrm{~s}$ at the annealing temperature listed in Table 1, and $45 \mathrm{~s}$ at $72^{\circ} \mathrm{C}$, with a final extension of $5 \mathrm{~min}$ at $72^{\circ} \mathrm{C}$. As the reference marker, a 10-bp DNA ladder (Invitrogen) was used to identify allele size.

The PCR products were resolved on $6 \%$ denaturing polyacrylamide gel using silver staining. The number of alleles and observed and expected heterozygosities were estimated with the Microsatellite Analyser program (Dieringer and Schlötterer, 2003), and tests for linkage disequilibrium and deviations from Hardy-Weinberg equilibrium were performed using Genepop 4.0 (Rousset, 2008).

\section{RESULTS AND DISCUSSION}

Of the 52 primer pairs developed, 24 microsatellite loci showed obvious polymorphism in the S. lessoniana population (see Table 1). The number of alleles at each locus ranged from 4 to 26 with an average of 13.25, and the observed and expected heterozygosities varied from 0.188 to 1.000 and 0.392 to 0.959 with an average of 0.675 and 0.852 , respectively. No linkage disequilibrium existed between pairs of loci. Significant departure from HardyWeinberg equilibrium was found at 10 loci (Sle02, Sle06, Sle07, Sle11, Sle15, Sle24, Sle25, Sle31, Sle36, and Sle41). These deviations may have been caused by the presence of null alleles at these loci (Pemberton et al., 1995). Genetic variability of these loci indicated their practicability as appropriate population genetic markers for the exploration and investigation of population genetic diversity in this species.

\section{ACKNOWLEDGMENTS}

Research supported by the grants from the National High Technology Research and Development Program of China (\#2007AA09Z433) and the Fundamental Research Funds for the Central Universities (\#201122005).

\section{REFERENCES}

Akasaki T, Nikaido M, Tsuchiya K, Segawa S, et al. (2006). Extensive mitochondrial gene arrangements in coleoid Cephalopoda and their phylogenetic implications. Mol. Phylogenet. Evol. 38: 648-658.

Dieringer D and Schlötterer C (2003). Microsatellite analyser (MSA): a platform independent analysis tool for large microsatellite data sets. Mol. Ecol. Notes 3: 167-169.

Gardner MG, Cooper SJB, Bull CM and Grant WN (1999). Isolation of microsatellite loci from a social lizard, Egernia stokesii, using a modified enrichment procedure. J. Hered. 90: 301-304.

Izuka T, Segawa S, Okutani T and Numachi K (1994). Evidence on the existence of three species in the oval squid Sepioteuthis lessoniana complex in Ishigaki island, Okinawa, southwestern Japan, by isozyme analyses. Jpn. J. Malacol. 53: 217-228.

Izuka T, Segawa S and Okutani T (1996). Biochemical study of the population heterogeneity and distribution of the oval squid Sepioteuthis lessoniana complex in southwestern Japan. Am. Malacol. Bull. 12: 129-135.

Knowlton N (1993). Sibling species in the sea. Ann. Rev. Ecol. Syst. 124: 189-216.

Li Q and Wan JM (2005). SSRHunter: development of a local searching software for SSR sites. Yi. Chuan 27: 808-810.

Pemberton JM, Slate J, Bancroft DR and Barrett JA (1995). Nonamplifying alleles at microsatellite loci: a caution for parentage and population studies. Mol. Ecol. 4: 249-252.

Rousset F (2008). Genepop'007: a complete re-implementation of the genepop software for Windows and Linux. Mol. Ecol. Resour. 8: 103-106.

Thorpe JP, Solé-Cava AM and Watts PC (2000). Exploited marine invertebrates: genetics and fisheries. Hydrobiol 420: 165-184. 
Triantafillos L and Adams M (2005). Genetic evidence that the northern calamary, Sepioteuthis lessoniana, is a species complex in Australian waters. ICES J. Mar. Sci. 62: 1665-1670.

Yeatman J and Benzie JAH (1994). Genetic structure and distribution of Photololigo spp. in Australia. Mar. Biol. 118: $79-87$. 